MS-TPI-92-20

\title{
Critical Wilson Lines in Toroidal Compactifications of Heterotic Strings*
}

\author{
Thomas Mohaupt \\ Institut für Theoretische Physik I, Universität Münster \\ Wilhelm-Klemm-Str. 9, D-4400 Münster, Germany \\ July 16, 1992
}

\begin{abstract}
Critical values of Wilson lines and general background fields for toroidal compactifications of heterotic string theories are constructed systematically using Dynkin diagrams.
\end{abstract}

*This work is part of a Ph.D. thesis in preparation at the science faculty of the university of Münster. 


\section{Introduction}

Toroidal compactifications [13], [21], [20] offer the simplest way to construct heterotic string theories in four space-time dimensions. Although they do not have a realistic spectrum they can be used as a starting point for the construction of other theories like toroidal orbifolds [5], [6], [22] or covariant lattice models [18], and some typical features of string compactifications can be studied easily because the inner or compactified degrees of freedom of the string can still be represented by a free conformal field theory. String compactifications and more generally non-minimal conformal field theories depend on continuous parameters, called moduli, which have been the object of many studies (see for example: [20], [7], [3], [4]). One is interested in the global geometry of moduli spaces and especially in its discrete symmetries under duality (or target space modular) transformations which in the string interpretation are probably connected to the existence of a minimal length scale [24], [10]. Another point of interest is the behaviour of the spectrum and symmetry group under continuous deformations and the existence of critical values with extended symmetry. This has been studied systematically for those moduli of toroidal compactifications which already appear in the bosonic case [8], [20], namely the metric $G_{i j}$ and the so called axionic background field $B_{i j}$. For the background gauge field (the so called Wilson lines) only particular examples [8] of critical values have been mentioned so far. The purpose of this paper is to study systematically Wilson lines and general background fields in toroidal compactifications. This is a first step to a better understanding of continuous background fields, especially Wilson lines, in more general situations. After reviewing the relevant facts about toroidal compactifications and Lie algebras I show in detail how the Lie algebra that is present in a toroidal compactification without Wilson lines and $B_{i j}$-field can be broken to any maximal regular subalgebra by a suitable choice of one Wilson line. This is done by implementing the extended Dynkin diagram formalism, which makes the procedure very transparent. The results are then generalized in two steps to general regular subalgebras

of maximal and nonmaximal rank. If the rank of the semisimple part of the Lie algebra is reduced, the method characterizes critical hypersurfaces instead of critical points. Then I investigate how the rank of the semisimple part of the gauge Lie algebra can be enlarged beyond 16. The generalization to general background fields does not only combine the old results about the 
$B_{i j}$-field (which are reproduced) with the new results about Wilson lines but also shows how the effects of the different background fields can "interfere". Finally the construction is translated into the language of shift vectors for rational background fields. In the last section I propose further applications to more general compactifications.

\section{Toroidal Compactifications with Background Fields}

The simplest way to get a four-dimensional (or more generally a D-dimensional, $D<10)$ theory from the ten-dimensional heterotic string is the compactification of six (or $d$ ) space dimensions on a torus [13]:

$$
\mathbf{R}^{10} \longrightarrow \mathbf{R}^{D} \times T^{d}
$$

where

$$
T^{d}=\frac{\mathbf{R}^{d}}{2 \pi \Lambda}, \quad d=10-D,
$$

and $\Lambda$ is a $d$-dimensional lattice with basis $\left\{\mathbf{e}_{i}\right\}$. Since this can also be interpreted as a compactification on the torus $\mathbf{R}^{d} / \mathbf{Z}^{d}$ in the presence of a constant background gravitational field, it is natural to admit nonzero constant vacuum expectation values for those fields, which are massless space-time scalars from the D-dimensional point of view. This leads to the Narain model [21], 20], a continuous family of string vacua parametrized by the vacuum expectation values of the symmetric and antisymmetric tensor field and of the gauge fields in the internal dimensions. A state of the compactified theory is not only labeled by its continuous space-time momentum $p^{\mu}, \mu=1, \ldots, D$, its oscillation state, and its gauge quantum numbers $p^{I}, I=1, \ldots, 16$, but also by its winding vector

$$
\mathbf{w}=w^{i} \mathbf{e}_{i} \in \Lambda,
$$

describing how the string wraps around the internal dimensions, and by its discrete internal momentum

$$
\mathbf{p}=p_{i} \mathbf{e}^{* i} \in \Lambda^{*} .
$$

$\left\{\mathbf{e}^{* i}\right\}$ is the standard basis of the dual lattice $\Lambda^{*}$ of $\Lambda$. The metrics of the two lattices are

$$
G^{i j}=\mathbf{e}^{* i} \cdot \mathbf{e}^{* j} \text { and } G_{i j}=\mathbf{e}_{i} \cdot \mathbf{e}_{j} \text {, where } G^{i j} G_{j k}=\mathbf{e}^{* i} \cdot \mathbf{e}_{k}=\delta_{k}^{i}
$$


The vectors which consist of the gauge quantum numbers $p^{I}$ of the ten dimensional theory are constrained to form a sixteen dimensional even selfdual euclidean lattice $\Gamma_{16}$ [13]. There are (up to isometries) only to two such lattices: the root lattice of the Lie algebra $E_{8} \oplus E_{8}$ and the sublattice $D_{16}^{(0)(s)}$ of the weight lattice of the Lie algebra $D_{16}$ that is generated by the roots and the spinor weights [2]. The corresponding gauge groups (of the ten dimensional theory) are $E_{8} \otimes E_{8}$ and $\operatorname{Spin}(32) / \mathbf{Z}_{2}$. All the internal quantum numbers of the D-dimensional theory can be combined into the left- and right-moving momenta, which form the momentum lattice $\Gamma \equiv \Gamma_{16+d ; d}$ :

$$
\left(\mathbf{p}_{L} ; \mathbf{p}_{R}\right)=\left(p_{L}^{I} \mathbf{e}_{I}+p_{L}^{i} \mathbf{e}_{i} ; p_{R}^{i} \mathbf{e}_{i}\right) \in \Gamma_{16+d ; d},
$$

where $\left\{\mathbf{e}_{I}\right\}$ is a basis of $\Gamma_{16}$, and [8]

$$
\begin{gathered}
p_{L}^{I}=p^{I}+A_{i}^{I} w^{i}, \\
p_{L}^{i}=\frac{1}{2} p^{i}-B_{k}^{i} w^{k}-\frac{1}{2} p^{J} A^{J i}-\frac{1}{4} A^{K i} A_{j}^{K} w^{j}+w^{i} \\
p_{R}^{i}=\frac{1}{2} p^{i}-B_{k}^{i} w^{k}-\frac{1}{2} p^{J} A^{J i}-\frac{1}{4} A^{K i} A_{j}^{K} w^{j}-w^{i}
\end{gathered}
$$

In these formulas $B_{i j}$ are the components of the antisymmetric tensor field and $A_{i}^{I}$ are the components of the background gauge field: There is one nontrivial gauge field configuration (called Wilson line) $\mathbf{A}_{i}=A_{i}^{I} \mathbf{e}_{I}$ for each generator of the fundamental group of the torus and all those fields must take values in a Cartan subalgebra (i.e. they all commute), that is in $\mathbf{R}^{16}$ [13], [20]. The dependence of $\Gamma$ on the lattice $\Lambda$ is implicit in the component notation in that the $i, j, \ldots$ indices are raised and lowered by the metric $G_{i j}=\mathbf{e}_{i} \cdot \mathbf{e}_{j}$ of the lattice $\Lambda$. The momentum lattice is, by construction, selfdual and even with respect to the signature $(+)^{16+d}(-)^{d}$; this is, as shown by Narain [21], equivalent to modular invariance of the theory. $G_{i j}, B_{i j}$ and $A_{i}^{I}$ parametrize all $S O(16+d ; d)$ - boosts of $\Gamma$ modulo proper rotations and, because there exists up to isometries only one self dual even lattice for each signature $8 n$, $n=1,2, \ldots$, one gets all models by varying these parameters] [20].

The vectors of the momentum lattice contain the gauge quantum numbers of the states. To each vector of the form

$$
\left(\mathbf{p}_{L} ; 0\right) \in \Gamma_{16+d ; d}, \quad \mathbf{p}_{L}^{2}=2,
$$

\footnotetext{
${ }^{1}$ Rotations are symmetries of the space of theories, and there are further, discrete identifications of theories due to duality 20].
} 
called a root ${ }^{2}$ of $\Gamma$, corresponds a charged, massless spin 1 state, that means a charged gauge boson. These states combine with $16+d$ uncharged gauge bosons, which are present for all choices of $\Gamma$, to form the adjoint representation of the gauge group of the model under consideration, which is of the form

$$
G=G^{(l)} \otimes U(1)^{16+d-l},
$$

where $G^{(l)}$ is a simply laced, semisimple Lie group of rank $l$ and $l, 0 \leq l \leq$ $16+d$ is the number of linear independent roots. If one sets $B_{i j}=0$ and $A_{i}^{I}=0$ ("compactification without background fields") the gauge groups are, according to the choice of the ten dimensional theory

$$
G=E_{8} \otimes E_{8} \otimes U(1)^{d} \text { or } G=\operatorname{Spin}(32) / \mathbf{Z}_{2} \otimes U(1)^{d} .
$$

Before we can study the effects of nonvanishing background fields on the gauge group systematically, we must recall some elements of Dynkins theory of subalgebras of semisimple Lie algebras.

\section{Mathematical preliminaries}

A semisimple Lie algebra $\mathrm{g}$ of rank $l$ can be decomposed into a Cartan subalgebra $\mathbf{h}$ and the one dimensional root spaces $\mathbf{g}_{\alpha}$, which are the eigenspaces with respect to the adjoint action of $\mathbf{h}$ on $\mathbf{g}$ :

$$
\mathbf{g}=\mathbf{h} \oplus \bigoplus_{\alpha \in \Sigma} \mathbf{g}_{\alpha}
$$

The root spaces are labeled by a finite set $\Sigma$ of l-dimensional vectors, whose components are the eigenvalues of the Cartan generators in the corresponding root space. From $\Sigma$ one can choose a set $\Pi=\left\{\alpha_{i}: i=1, \ldots, l\right\}$ of simple roots, from which all roots and the Lie algebra itself can be reconstructedf

\footnotetext{
${ }^{2}$ This useful terminology was introduced in the context of the covariant lattice construction 18$]$.

${ }^{3}$ The material presented here can be found in the book by Cahn [1], as far as semisimple Lie algebras are concerned. A good reference for general Lie algebras is [14]. A useful collection of data of simple Lie algebras is given in appendix $\mathrm{F}$ of the book [2].

${ }^{4}$ The same amount of information is encoded also in the Cartan matrix and in the Dynkin diagram.
} 
A subalgebra $\mathbf{g}^{\prime}$ of $\mathbf{g}$ is called regular, if it respects this decomposition in the sense that its decomposition fulfills $\mathbf{h}^{\prime} \subset \mathbf{h}$ and $\Sigma^{\prime} \subset \Sigma$. Non regular subalgebras will be ignored in the following, because the method that I will describe in the next section works by reducing the set of roots and so only gives regular subalgebras. As the rank of the gauge group is fixed (to be $16+d)$ in toroidal compactifications we can restrict ourselves to regular subalgebras of maximal rank. In order to construct subalgebras in a systematic way, one defines $\mathbf{g}^{\prime}$ to be a maximal subalgebra of $\mathbf{g}$, if

$$
\mathrm{g}^{\prime} \subset \tilde{\mathrm{g}} \subset \mathrm{g} \Longrightarrow \tilde{\mathrm{g}}=\mathrm{g}^{\prime} \text { or } \tilde{\mathrm{g}}=\mathrm{g}
$$

for all subalgebras $\tilde{\mathrm{g}}$. This notion needs further refinement because a maximal subalgebra of a semisimple Lie algebra is not necessarily semisimple. A "maximal semisimple subalgebra" is thus a subalgebra which is maximal among the semisimple subalgebras. The only non semisimple subalgebras that will appear in the following are of the form semisimple $\oplus$ abelian, because other types of subalgebras cannot be realized?

Dynkin has given a (not entirely correct, see below) rule to construct all maximal semisimple subalgebras of a simple Lie algebra. (This extends to semisimple Lie algebras because they are direct sums of simple ones.) The rule can be stated diagrammatically by saying that one has to remove, in all possible ways, one dot from the extended Dynkin diagram of the simple Lie algebra $\mathbf{g}$. This means, in algebraic terms, that one chooses a system of simple roots (which can be represented by a Dynkin diagram), then extends this by the lowest root (thus extending the Dynkin diagram) and finally deletes one root. The resulting set is the system of simple roots of a maximal rank, maximal semisimple regular subalgebra of $\mathbf{g}$. (There are five exceptional cases in which the subalgebra constructed this way is not maximal. See (52)) This subalgebra does not need to be proper, but one gets all maximal semisimple regular subalgebras and by iteration of the procedure all maximal rank, regular semisimple subalgebras ?

\footnotetext{
${ }^{5}$ These other types of subalgebras have root systems which do not contain the negatives of some roots, so they have an nilpotent part. A representation of such an algebra cannot be constructed by the lattice technique used in toroidal compactifications, because the negative of a lattice vector is always a lattice vector itself, and also violates CPT-Invariance, because some antiparticles are missing.

${ }^{6}$ It can also happen that one gets different but conjugated subalgebras while generating all these algebras. The uniqueness problem will be discussed later.
} 
In order to get the regular semisimple subalgebras of non maximal rank, one has to combine this rule with a second one [1], [19]: Removing $k$ dots, $0<k<l$ from the Dynkin diagram of a simple Lie algebra of rank $l$ gives the Dynkin diagram of a regular semisimple subalgebra of rank $l-k$, and all regular semisimple subalgebras of this rank can be constructed by removing, in all possible ways, $k$ dots from all rank $l$ regular semisimple subalgebrast?

In our application to the Narain model the total rank of the gauge group is fixed, as explained above. So we will only be able to reduce the rank of the semisimple part by removing roots, while the Cartan subalgebra is fixed, thus giving regular subalgebras of the type semisimple $\oplus$ abelian.

\section{Breaking to maximal Subalgebras by one Wilson line}

\subsection{Formulation of the symmetry breaking problem}

The discussion of symmetry breaking by Wilson lines starts naturally with a compactification on a lattice $\Lambda$ with "no background fields" 8 , that is with $B_{i j}=0$ and $A_{i}^{I}=0$. The momentum lattice $\Gamma$ is then of the form

$$
\Gamma_{16+6 ; 6}=\Gamma_{16} \oplus \Gamma_{6 ; 6},
$$

where $\Gamma_{16}$ is one of the two sixteen-dimensional euclidean even selfdual lattices mentioned above and $\Gamma_{6 ; 6}$ is a twelve-dimensional lorentzian even selfdual lattice with zero signature, which is orthogonal to $\Gamma_{16}$. The momentum lattice has a natural basis [8] that is given by the basis of $\Lambda, \Lambda^{*}$ and $\Gamma_{16}$ :

$$
\begin{gathered}
\mathbf{k}^{i}=\left(0, \frac{1}{2} \mathbf{e}^{* i} ; \frac{1}{2} \mathbf{e}^{* i}\right) \\
\overline{\mathbf{k}}_{i}=\left(0, \mathbf{e}_{i} ;-\mathbf{e}_{i}\right) \\
\mathbf{l}_{A}=\left(\mathbf{e}_{A}, 0 ; 0\right)
\end{gathered}
$$

\footnotetext{
${ }^{7}$ Isomorphic subalgebras need not be conjugated, if the Lie algebra has outer automorphisms 19.

${ }^{8}$ Remind that the specification of the background metric $G_{i j}$ is equivalent to the specification of the lattice $\Lambda$.
} 
The nonvanishing (lorentzian) scalar products between these basis vectors are:

$$
\mathbf{k}^{i} \overline{\mathbf{k}}_{j}=\delta_{j}^{i}, \quad \mathbf{l}_{A} \mathbf{l}_{B}=g_{A B}^{(16)}
$$

where $g_{A B}^{(16)}$ is the metric of $\Gamma_{16}$. The choice of this parametrization is very useful, because the switching on of background fields corresponds to an isometry of the momentum lattice and thus preserves the relations (17). What changes is the relation between the momentum lattice and the other lattices? [8]:

$$
\begin{gathered}
\mathbf{k}^{\prime i}=\left(0, \frac{1}{2} \mathbf{e}^{* i} ; \frac{1}{2} \mathbf{e}^{* i}\right) \\
\overline{\mathbf{k}}_{i}^{\prime}=\left(\mathbf{A}_{i},-B_{j i} \mathbf{e}^{* j}-\frac{1}{4}\left(\mathbf{A}_{j} \cdot \mathbf{A}_{i}\right) \mathbf{e}^{* j}+\mathbf{e}_{i} ;-B_{j i} \mathbf{e}^{* j}-\frac{1}{4}\left(\mathbf{A}_{j} \cdot \mathbf{A}_{i}\right) \mathbf{e}^{* j}-\mathbf{e}_{i}\right) \\
\mathbf{l}_{A}=\left(\mathbf{e}_{A},-\frac{1}{2}\left(\mathbf{e}_{A} \cdot \mathbf{A}_{i}\right) \mathbf{e}^{* i} ;-\frac{1}{2}\left(\mathbf{e}_{A} \cdot \mathbf{A}_{i}\right) \mathbf{e}^{* i}\right)
\end{gathered}
$$

The vectors $\mathbf{A}_{i}=A_{i}^{I} \mathbf{e}_{I}$ are called Wilson lines. In order to learn about the patterns of symmetry breaking it is useful to study the effect of one single Wilson line by setting $B_{i j}=0, \mathbf{A}_{i}=0, i \neq 1$, and taking the one direction of $\Lambda$, to which the Wilson line is assigned, to be orthogonal to the others. The momentum lattice is then in general of the form $\Gamma_{17 ; 1} \oplus \Gamma_{5 ; 5}$. We can now analyze how the gauge group depends on the sixteen parameters $A_{1}^{I}$. If also $A_{1}^{I}=0$, the roots of the momentum lattice are given by the basis vectors $l_{A}=\left(\mathbf{e}_{A}, 0 ; 0\right), A=1, \ldots, 16$ : When $\Gamma_{16}$ is the $E_{8} \oplus E_{8}$ root lattice, the basis vectors $\mathbf{e}_{A}$ are automatically a set of simple roots $\Pi=\left\{\alpha_{A}=\mathbf{e}_{A}\right\}$ of the corresponding Lie algebra and their integer linear combinations of norm squared 2 are the roots. When $\Gamma_{16}=D_{16}^{(o)(s)}$ the basis consists of all but one of the simple roots of $D_{16}$ plus one spinor weight vector, and the missing simple root is given by an integer linear combination [2]:

$$
\begin{gathered}
\mathbf{e}_{1}=\frac{1}{2} \sum_{A=1}^{8} \alpha_{2 A-1}, \quad \mathbf{e}_{A}=\alpha_{A}, A=2, \ldots, 16 \\
\alpha_{1}=2 \mathbf{e}_{1}-\sum_{A=1}^{7} \mathbf{e}_{2 A+1}
\end{gathered}
$$

\footnotetext{
${ }^{9}$ In canonical operator quantization a shift of background fields induces a shift of the canonical momentum relative to the kinetic momentum 20].
} 
As we are at the moment only interested in the gauge group, but not in the complete spectrum, we will only consider the sublattice generated by the simple roots. So we find that in both cases there is a set of vectors

$$
\widehat{\alpha}_{A}=\left(\alpha_{A}, 0 ; 0\right)
$$

which may be called simple roots of $\Gamma$ because they are a generating set for the roots of $\Gamma$ (which, remember, correspondent precisely to the charged gauge bosons). If we now switch on our Wilson line $\mathbf{A}_{1}$ these roots are mapped to

$$
\widehat{\alpha}_{A}^{\prime}=\left(\alpha_{A},-\frac{1}{2}\left(\alpha_{A} \cdot \mathbf{A}_{1}\right) \mathbf{e}^{* 1} ;-\frac{1}{2}\left(\alpha_{A} \cdot \mathbf{A}_{1}\right) \mathbf{e}^{* 1}\right)=\widehat{\alpha}_{A}-\left(\alpha_{A} \cdot \mathbf{A}_{1}\right) \mathbf{k}^{1}
$$

Since $\mathbf{k}^{1} \equiv \mathbf{k}^{\prime 1}$ and the momentum lattice is selfdual for all values of the background fields, we deduce that $\widehat{\alpha}_{A}$ is in the new momentum lattice $\Gamma^{\prime}$ if and only if $\alpha_{A} \cdot \mathbf{A}_{1}$ is an integer ${ }^{\mathbb{T}}$. Combining this observation with Dynkins theorem on maximal regular semisimple maximal rank subalgebras we can formulate the conditions the Wilson line $\mathbf{A}_{1}$ must obey in order to break the Lie algebra $\mathbf{g}$ of the gauge group to such an subalgebra: Obviously we must project out one of the simple roots, while keeping the others and the lowest root $-\alpha_{h}$. We can now state the symmetry breaking problem:

If the Wilson line $\mathbf{A}_{1}$ shall break the gauge Lie algebra to the maximal subalgebra that is defined by removing the I-th dot from the extended Dynkin diagram, then it must fulfill:

$$
\begin{gathered}
\alpha_{I} \cdot \mathbf{A}_{1} \notin \mathbf{Z} \\
\alpha_{A} \cdot \mathbf{A}_{1} \in \mathbf{Z}, \quad(A \neq I) \\
\alpha_{h} \cdot \mathbf{A}_{1} \in \mathbf{Z}
\end{gathered}
$$

\subsection{The standard solution of the symmetry breaking problem}

The next step is to show, that these conditions can be fulfilled for arbitrary I. This is somewhat trivial, because the equations above mean nothing but

\footnotetext{
${ }^{10}$ Note that all vectors in $\Gamma^{\prime}$ must have mutual integer scalar products and that $\widehat{\alpha}_{A}$ and $\widehat{\alpha}_{A}^{\prime}$ cannot be in the same selfdual lattice, if this condition is not fulfilled.
} 
$\mathbf{A}_{1}$ being a weight vector of the subalgebra but not of the algebra, but it is instructive and useful to construct an explicit solution. The symmetry breaking problem (23) - (25) will be solved for an arbitrary simple Lie algebra of arbitrary rank $l$. This extends trivially to the semisimple case. It is not necessary to specify the Lie algebra, and the technique used here will be useful in the following.

Let $\mathbf{g}$ be a simple simply laced Lie algebra of rank $l$ and with simple roots $\left\{\alpha_{1}, \ldots, \alpha_{l}\right\}$ and lowest root $-\alpha_{h}$ and let $\mathbf{g}^{\prime}$ be the regular maximal rank semisimple subalgebra with simple roots

$$
\left\{\beta_{1}, \ldots, \beta_{l}\right\}=\left\{\alpha_{1}, \ldots, \alpha_{I-1},-\alpha_{h}, \alpha_{I+1}, \ldots, \alpha_{l}\right\}
$$

The equations (24) and (25) imply that $\mathbf{A}_{1}$ is a weight vector of $\mathbf{g}^{\prime}$ and thus is an integer linear combination of the fundamental weight vectors $\beta_{A}^{*}$ of $\mathbf{g}^{\prime}$ :

$$
\mathbf{A}_{1}=\sum_{A=1}^{l} a^{A} \beta_{A}^{*}, \quad a^{A} \in \mathbf{Z}
$$

The highest root $\alpha_{h}$ of $\mathbf{g}$ is a (known, see [2]) integer linear combination of the simple roots of $\mathbf{g}$ :

$$
\alpha_{h}=\sum_{A=1}^{l} k^{A} \alpha_{A}, \quad k^{A} \in \mathbf{Z}, k^{A} \geq 0
$$

These equations can be used to express the simple roots of $\mathbf{g}$ as rational linear combinations of the simple roots of $\mathbf{g}^{\prime}$ :

$$
\begin{gathered}
\alpha_{I}=\frac{1}{k^{I}}\left(\alpha_{h}-\sum_{A \neq I} k^{A} \alpha_{A}\right)=\frac{1}{k^{I}}\left(\beta_{I}-\sum_{A \neq I} k^{A} \beta_{A}\right) \\
\alpha_{A}=\beta_{A}, \text { for } A \neq I
\end{gathered}
$$

If $k^{I}=1, \alpha_{I}$ is a root of $\mathbf{g}^{\prime}$ and the subalgebra is not a proper one: $\mathbf{g}^{\prime}=\mathbf{g}$. This corresponds to those cases in Dynkins method where by removing a dot from the extended Dynkin diagram one gets the original Dynkin diagram. If $k^{I}>1$ the equations (24) and (25) are identically satisfied and it remains to check equation (23). Since by definition $\beta_{A}^{*} \cdot \beta_{B}=\delta_{A B}$ we get by substituting (27) into (23):

$$
\alpha_{I} \cdot \mathbf{A}_{1}=-\frac{1}{k^{I}} a^{I}-\sum_{A \neq I} \frac{k^{A} a^{A}}{k^{I}}
$$


Choosing simply $\mathbf{A}_{1}=\beta_{I}^{*}$, i.e. $a^{A}=\delta_{A I}$ we get

$$
\alpha_{I} \cdot \mathbf{A}_{I}=-\frac{1}{k^{I}} \notin \mathbf{Z}
$$

because $k^{I}>1$. This solution is the most natural one because $\mathbf{A}_{1}$ is chosen to be the only fundamental weight vector of the subalgebra that is not a weight of $\mathbf{g}$. Therefore

$$
\mathbf{A}_{1}=\beta_{I}^{*}
$$

will be called the standard solution of the symmetry breaking problem. The considerations of this section have shown that Dynkins formalism can be implemented in the Narain model in a simple and natural way.

\subsection{All solutions of the symmetry breaking problem}

The next question to be investigated is how many other solutions of the equations (23) - 25) exist. As these equations are equivalent to the statement, that $\mathbf{A}_{1}$ is a weight vector of $\mathbf{g}^{\prime}$ but not of $\mathbf{g}$, the general solution can be parametrized in the following way:

$$
\mathbf{A}_{1}=\beta_{I}^{*}+\mathbf{v}, \text { where } \mathbf{v} \in \Gamma_{W}\left(\mathbf{g}^{\prime}\right)
$$

Let now $\Gamma^{\prime}$ be the lattice corresponding to the standard choice $\mathbf{v}=0$ of the Wilson line, whereas $\Gamma^{\prime \prime}$ is some other solution of the symmetry breaking problem with $\mathbf{v} \neq 0$. These lattices can be compared most easily by looking at there basis vectors. In the $E_{8} \oplus E_{8}$ case basis for $\Gamma^{\prime}$ and $\Gamma^{\prime \prime}$ are given by

$$
\begin{gathered}
\overline{\mathbf{k}}_{1}^{\prime}=\left(\beta_{I}^{*},-\frac{1}{2}\left(\beta_{I}^{*}\right)^{2} \frac{1}{2} \mathbf{e}^{* 1}+\mathbf{e}_{1} ;-\frac{1}{2}\left(\beta_{I}^{*}\right)^{2} \frac{1}{2} \mathbf{e}^{* 1}-\mathbf{e}_{1}\right) \\
\overline{\mathbf{k}}_{1}^{\prime \prime}=\left(\beta_{I}^{*}+\mathbf{v},-\frac{1}{2}\left(\left(\beta_{I}^{*}+\mathbf{v}\right)^{2}\right) \frac{1}{2} \mathbf{e}^{* 1}+\mathbf{e}_{1} ;-\frac{1}{2}\left(\left(\beta_{I}^{*}+\mathbf{v}\right)^{2}\right) \frac{1}{2} \mathbf{e}^{* 1}-\mathbf{e}_{1}\right) \\
\widehat{\alpha}_{I}^{\prime}=\left(\alpha_{I},-\left(\alpha_{I} \cdot \beta_{I}^{*}\right) \frac{1}{2} \mathbf{e}^{* 1} ;-\left(\alpha_{I} \cdot \beta_{I}^{*}\right) \frac{1}{2} \mathbf{e}^{* 1}\right) \\
\widehat{\alpha}_{I}^{\prime \prime}=\left(\alpha_{I},-\left(\alpha_{I} \cdot \beta_{I}^{*}+\alpha_{I} \cdot \mathbf{v}\right) \frac{1}{2} \mathbf{e}^{* 1} ;-\left(\alpha_{I} \cdot \beta_{I}^{*}+\alpha_{I} \cdot \mathbf{v}\right) \frac{1}{2} \mathbf{e}^{* 1}\right) \\
\widehat{\alpha}_{A}=\widehat{\alpha}_{A}^{\prime}=\widehat{\alpha}_{A}^{\prime \prime}=\left(\alpha_{A}, 0 ; 0\right), \text { for } A \neq I
\end{gathered}
$$




$$
\mathbf{k}^{\prime \prime i}=\mathbf{k}^{\prime i}=\mathbf{k}^{i} \text { for all } i, \quad \overline{\mathbf{k}}_{i}^{\prime \prime}=\overline{\mathbf{k}}_{i}^{\prime}=\overline{\mathbf{k}}_{i} \text { for } i \neq 1
$$

(The unprimed vectors are the standard basis (16) of the lattice without background fields, $\Gamma$.) We also need the images of the highest root $\widehat{\alpha}_{h}$ of $\Gamma$ in $\Gamma^{\prime}$ and $\Gamma^{\prime \prime}$ :

$$
\begin{gathered}
\widehat{\alpha}_{h}^{\prime}=\widehat{\alpha}_{h}+\left(-\alpha_{h} \cdot \beta_{I}^{*}\right) \mathbf{k}^{1}=\widehat{\alpha}_{h}+\mathbf{k}^{1} \\
\widehat{\alpha}_{h}^{\prime \prime}=\widehat{\alpha}_{h}+\left(1-\alpha_{h} \cdot \mathbf{v}\right) \mathbf{k}^{1}, \text { where }-\alpha_{h} \cdot \mathbf{v} \in \mathbf{Z}
\end{gathered}
$$

In the $D_{16}$ case there is one basis vector $l_{1}^{\prime \prime}$ (see (19)) in the 16-dimensional sector that is not a root, but constructed from a spinor weight. But I will analyze the $E_{8} \oplus E_{8}$ case, where the basis of the lattices $\Gamma^{\prime}$ and $\Gamma^{\prime \prime}$ only differ by two vectors $\overline{\mathbf{k}}_{1}^{\prime \prime}$ and $\widehat{\alpha}_{I}^{\prime \prime}$. The arguments given below can be extended to the more complicated case by treating $l_{1}^{\prime \prime}$ the same way as $\widehat{\alpha}_{I}^{\prime \prime}$ will be dealt with below.

In order to make the analysis as simple as possible, let us assume for the moment that $\mathbf{v}$ is a weight vector of $\mathbf{g}$, which implies $\alpha_{I} \cdot \mathbf{v} \in \mathbf{Z}$. Then $\widehat{\alpha}_{I}^{\prime \prime}$ and $\widehat{\alpha}_{I}^{\prime}$ are in $\Gamma^{\prime} \cap \Gamma^{\prime \prime}$ and we must only care about $\overline{\mathbf{k}}_{1}^{\prime \prime}$. If $\mathbf{v}$ is even in the root lattice of $\mathbf{g}^{\prime}$ we can expand it

$$
\widehat{\mathbf{v}} \equiv(\mathbf{v}, \mathbf{0} ; \mathbf{0})=\sum_{A=1}^{l} v^{A}\left(\beta_{A}, \mathbf{0} ; \mathbf{0}\right) \equiv \sum_{A=1}^{l} v^{A} \widehat{\beta}_{A}, \text { where } v^{A} \in \mathbf{Z},
$$

implying $\widehat{\mathbf{v}} \in \Gamma^{\prime \prime}$. As $\mathbf{v}$ is in the root lattice and $\beta_{I}^{*}$ is a weight vector of $\mathbf{g}^{\prime}$ it follows $\mathbf{v}^{2} \in 2 \mathbf{Z}, \mathbf{v} \cdot \beta_{I}^{*} \in \mathbf{Z}$ and

$$
\overline{\mathbf{k}}_{1}^{\prime \prime}-\overline{\mathbf{k}}_{1}^{\prime}=\widehat{\mathbf{v}}-\left(\beta_{I}^{*} \cdot \mathbf{v}+\frac{1}{2} \mathbf{v}^{2}\right) \mathbf{k}^{1}
$$

This difference being in $\Gamma^{\prime \prime}$ implies $\overline{\mathbf{k}}_{1}^{\prime} \in \Gamma^{\prime \prime}$. As all basis vectors of $\Gamma^{\prime \prime}$ are in $\Gamma^{\prime}$ and vice versa both lattices are equal. If we want to construct a physically different solution of the symmetry breaking problem, we must choose $\mathbf{v} \notin$ $\Gamma_{R}\left(\mathbf{g}^{\prime}\right)$ and solutions differing by elements of $\Gamma_{R}\left(\mathbf{g}^{\prime}\right)$ are identical. As $\mathbf{v}$ must be in the weight lattice of $\mathbf{g}^{\prime}$ we can conclude: If $\mathbf{v}$ is a weight vector of $\mathbf{g}$, then the inequivalent solutions of the symmetry breaking problem are parametrized by the elements of the finite factor group

$$
\Gamma_{W}\left(\mathbf{g}^{\prime}\right) / \Gamma_{R}\left(\mathbf{g}^{\prime}\right)
$$


This group is well known in the theory of Lie groups and Lie algebras because it is (isomorphic to) the group of conjugacy classes of representations of $\mathbf{g}^{\prime}$ and the center of the universal linear group $\exp \left(\mathbf{g}^{\prime}\right)$ of $\mathbf{g}^{\prime}$ [11], [2], [18].

Let us now have a look on $\widehat{\alpha}_{I}^{\prime \prime}$ and relax the condition on $\alpha_{I} \cdot \mathbf{v}$. Since

$$
\widehat{\alpha}_{I}^{\prime \prime}=\widehat{\alpha}-\left(\alpha_{I} \cdot \beta_{I}^{*}+\alpha_{I} \cdot \mathbf{v}\right) \mathbf{k}^{1}=\widehat{\alpha}_{I}^{\prime}-\left(\alpha_{I} \cdot \mathbf{v}\right) \mathbf{k}^{1},
$$

$\alpha_{I} \cdot \mathbf{v} \notin \mathbf{Z}$, which is equivalent to $\mathbf{v} \notin \Gamma_{W}(\mathbf{g})$, implies $\widehat{\alpha}_{I}^{\prime \prime} \notin \Gamma^{\prime}$ and $\Gamma^{\prime \prime} \neq \Gamma^{\prime}$. But as always $\mathbf{v} \in \Gamma_{W}\left(\mathbf{g}^{\prime}\right)$ and (29), no summation of I)

$$
k^{I} \alpha_{I}=-\beta_{I}-\sum_{A \neq I} k^{A} \beta_{A} \in \Gamma_{R}\left(\mathbf{g}^{\prime}\right)
$$

we have

$$
\alpha_{I} \cdot \mathbf{v}=\frac{m}{k^{I}} \bmod \mathbf{Z}, \quad \exists m \in\left\{1, \ldots, k^{I}\right\}
$$

As both $\alpha_{I}$ and $\mathbf{v}$ are weights of $\mathbf{g}^{\prime}$ their scalar product modulo integers is characterized by their conjugacy classes [18]. Combining this with the previous result on the dependence of $\Gamma^{\prime \prime}$ on $\overline{\mathbf{k}}_{1}^{\prime \prime}$ we conclude: The inequivalent solutions of the symmetry breaking problem are parametrized by the elements of the finite factor group

$$
\Gamma_{W}\left(\mathbf{g}^{\prime}\right) / \Gamma_{R}\left(\mathbf{g}^{\prime}\right)
$$

A further interesting observation, which will be useful later, is that the case $m=1$ in equation (48) is special. We then have:

$$
\alpha_{I} \cdot \mathbf{v}=\frac{1}{k^{I}} \Rightarrow \alpha_{I} \cdot \beta_{I}^{*}+\alpha_{I} \cdot \mathbf{v}=0 \Rightarrow \widehat{\alpha}_{I} \in \Gamma^{\prime \prime}
$$

That means that all roots of $\mathbf{g}$ are in $\Gamma^{\prime \prime}$ and the algebra of symmetries realized is in fact $\mathbf{g}$. The condition (23), which makes sure that $\alpha_{I}$ is projected out, is not fulfilled, because $\mathbf{A}_{1}=\beta_{I}^{*}+\mathbf{v} \in \Gamma_{W}(\mathbf{g})$. If $\mathbf{A}_{1} \in \Gamma_{R}(\mathbf{g})$ the basis vectors of $\Gamma^{\prime \prime}$ are all in $\Gamma$ implying $\Gamma^{\prime \prime}=\Gamma$. If not, the lattices have the same roots but are different, that means they describe theories with identical gauge group but different spectra. A necessary condition to break $\mathbf{g}$ to $\mathbf{g}^{\prime}$ is

$$
\mathbf{A}_{1} \in \Gamma_{W}\left(\mathbf{g}^{\prime}\right)-\Gamma_{W}(\mathbf{g})
$$

As $\mathbf{g}^{\prime}$ is a maximal subalgebra and this choice defines a proper subalgebra of $\mathrm{g}$ that contains $\mathbf{g}^{\prime}$, it is also sufficient. 
We have also to remember that there are cases where Dynkins rule does not give a maximal subalgebras. But there are only the five following exceptions [12], [1] where the algebra on the left is the would-be-maximal subalgebram:

$$
\begin{array}{cccccc}
A_{3} \oplus A_{3} \oplus A_{1} & \subset & D_{6} \oplus A_{1} & \subset & E_{7} \\
A_{3} \oplus D_{5} & \subset & D_{8} & \subset & E_{8} \\
A_{1} \oplus A_{2} \oplus A_{5} & \subset & A_{2} \oplus E_{6} & \subset & E_{8} \\
A_{1} \oplus A_{7} & \subset & A_{1} \oplus E_{7} & \subset & E_{8} \\
A_{3} \oplus A_{1} & \subset & B_{4} & \subset & F_{4}
\end{array}
$$

The fifth case is concerned with non simply laced Lie algebras and cannot appear in our context. The other cases are relevant to the breaking of $E_{8}$. They will be discussed in the next section where we will analyze how to break the gauge algebra to a general semisimple maximal rank subalgebra.

\section{Maximal rank semisimple subalgebras}

Every maximal rank semisimple subalgebra of $\mathbf{g}$ will appear in a chain of maximal semisimple maximal rank subalgebras:

$$
\mathbf{g} \supset \mathbf{g}^{(1)} \supset \mathbf{g}^{(2)} \supset \cdots
$$

As the root and weight lattices of simply laced Lie algebras satisfy

$$
\Gamma_{R} \subset \Gamma_{W}=\Gamma_{R}^{*}
$$

each chain (53) of maximal subalgebras induces a chain of lattices:

$$
\cdots \Gamma_{R}\left(\mathbf{g}^{(2)}\right) \subset \Gamma_{R}\left(\mathbf{g}^{(1)}\right) \subset \Gamma_{R}(\mathbf{g}) \subset \Gamma_{W}(\mathbf{g}) \subset \Gamma_{W}\left(\mathbf{g}^{(1)}\right) \subset \Gamma_{W}\left(\mathbf{g}^{(2)}\right) \cdots
$$

Then every choice

$$
\mathbf{A}_{1} \in \Gamma_{W}\left(\mathbf{g}^{(n)}\right)-\Gamma_{W}\left(\mathbf{g}^{(n-1)}\right)
$$

of the Wilson line will break $\mathbf{g}$ precisely to the subalgebra $\mathbf{g}^{(n)}$, if we assume for the moment that $\mathbf{g}^{(n)}$ only appears in one such chain. To prove this note first that for all roots $\alpha$ of $\mathbf{g}^{(n)}$

$$
\mathbf{A}_{1} \cdot \alpha \in \mathbf{Z} \Longrightarrow \widehat{\alpha} \in \Gamma=\Gamma_{17 ; 1} \oplus \Gamma_{5 ; 5}
$$

\footnotetext{
11 Note that there is an apparent misprint in the book [1].
} 
because a vector is in the selfdual momentum lattice if and only if it has integer scalar products with all basis vectors. This implies that all roots of $\mathbf{g}^{(n)}$ are roots of the momentum lattice and $\mathbf{g}^{(n)}$ is contained in the gauge Lie algebra. If on the other hand $\beta$ is a root of $\mathbf{g}^{(n-1)}$ that is not a root of $\mathbf{g}^{(n)}$, assume $\mathbf{A}_{1} \cdot \beta \in \mathbf{Z}$, such that $\widehat{\beta} \in \Gamma$. By linearity $\Gamma$ then contains the root system of a Lie algebra that is bigger than $\mathbf{g}^{(n)}$ and a subalgebra of $\mathbf{g}^{(n-1)}$. By maximality this Lie algebra must be $\mathbf{g}^{(n-1)}$ itself, but this is an contradiction because $\mathbf{A}_{1}$ must by construction have a non integer scalar product with at least one root of $\mathbf{g}^{(n-1)}$. Therefore we can conclude

$$
\mathbf{A}_{1} \cdot \beta \notin \mathbf{Z} \Longrightarrow \widehat{\beta} \notin \Gamma
$$

This shows how to realize an arbitrary maximal rank subalgebra, as long as it does not belong simultaneously to two different chains of maximal subalgebras. If some subalgebra is maximal in more than two subalgebras of $\mathbf{g}$ one has to exclude all their weight lattices in (56). The cases in which Dynkins rule fails are similar in that one must check whether the Wilson line breaks $\mathrm{g}$ to the would-be-maximal subalgebra or to the maximal subalgebra. But this is most easily understood by an example, which may also be useful to illustrate other aspects of the formalism.

Example: Let us take the $E_{8} \oplus E_{8}$ model as our example and ignore the second $E_{8}$. The vectors $\widehat{\alpha}_{A}, A=1, \ldots, 8$, where $\alpha_{A}$ are the simple roots of $E_{8}$, are basis vectors of the momentum lattice. Looking at the extended Dynkin diagram, we find that the lowest root is $-\alpha_{7}^{*}$. It can be expanded in the simple root basis using the inverse of the Cartan matrix $C\left(E_{8}\right)$ of $E_{8}$ :

$$
\begin{gathered}
-\alpha_{7}^{*}=-\sum_{A=1}^{8} k^{A} \alpha_{A}=-\sum_{A=1}^{8} C\left(E_{8}\right)_{7 A}^{-1} \alpha_{A} \\
=-2 \alpha_{1}-4 \alpha_{2}-6 \alpha_{3}-5 \alpha_{4}-4 \alpha_{5}-3 \alpha_{6}-2 \alpha_{7}-3 \alpha_{8}
\end{gathered}
$$

In order to break $E_{8}$ to the subalgebra $E_{7} \oplus A_{1}$, we have to remove $\alpha_{7}$. To choose the Wilson line we write down the simple roots of the subalgebra:

$$
E_{7}: \beta_{A}=\alpha_{A}, A=1, \ldots, 6, \beta_{7}=\alpha_{8}, \quad A_{1}: \beta=-\alpha_{7}^{*}
$$

\footnotetext{
${ }^{12}$ The conventions for roots used here are the same as in the book by Cornwell.
} 
The standard choice (33) for the Wilson line is then the dual of the new simple root $\beta$, where "dual" refers to the subalgebra $E_{7} \oplus A_{1}$ :

$$
\mathbf{A}_{1}=\beta^{*}=\frac{\beta}{\beta \cdot \beta}
$$

Working out the scalar products between the simple roots and this Wilson line shows that in fact only

$$
\beta^{*} \cdot \alpha_{7}=\frac{-\alpha_{7}^{*} \cdot \alpha_{7}}{\alpha_{7}^{*} \cdot \alpha_{7}^{*}}=\frac{-1}{2}
$$

is non-integer. When we now look at the extended Dynkin diagram of $E_{7}$ we find that the lowest root is $-\beta_{1}^{*}$ and that we can break $E_{7} \oplus A_{1}$ to $A_{7} \oplus A_{1}$ by removing the seventh root of $E_{7}$. The basis vectors of the subalgebra are:

$$
A_{7}: \gamma_{1}=-\beta_{1}^{*}=\sum_{A=1}^{7} C\left(E_{7}\right)_{1 A}^{-1} \beta_{A}, \quad \gamma_{A}=\beta_{A-1}, A=2, \ldots, 7, \quad A_{1}: \beta
$$

and the standard Wilson line is therefore

$$
\mathbf{A}_{1}=\beta^{*}+\gamma_{1}^{*}, \quad \gamma_{1}^{*}=\sum_{A=1}^{7} C\left(A_{7}\right)_{1 A}^{-1} \gamma_{A}
$$

This subalgebra is one of the exceptions to Dynkins rule because it is not maximal in $E_{8}$ but can be constructed directly from the extended Dynkin diagram of $E_{8}$ by removing the second root ${ }^{[3}[1],[12$. Applying the procedure to this case we choose a basis

$$
A_{7}: \gamma_{1}^{\prime}=\alpha_{8}, \gamma_{A}^{\prime}=\alpha_{A+1}, A=2, \ldots, 6, \gamma_{7}^{\prime}=-\alpha_{7}^{*}, \quad A_{1}: \beta^{\prime}=\alpha_{1}
$$

and the standard Wilson line

$$
\mathbf{A}_{1}=\left(\gamma_{7}^{\prime}\right)^{*}=\sum_{A=1}^{7} C\left(A_{7}\right)_{7 A}^{-1} \gamma_{A}^{\prime}
$$

But since $A_{7} \oplus A_{1}$ is not maximal in $E_{8}$ we do not know whether some extra roots survive the projection and extend the root system to the one of $E_{7} \oplus A_{1}$. These extra roots must then come from the weight $-\left(\gamma_{4}^{\prime}\right)^{*}$ of $E_{7}$ that extends

\footnotetext{
13 These two subalgebras are isomorphic and conjugated by an inner automorphism 12 .
} 
the Dynkin diagram of $A_{7}$ to the extended Dynkin diagram of $E_{7}$ the The vector $\left(\widehat{\gamma}_{4}^{\prime}\right)^{*}$ is in the momentum lattice if and only if $\left(\gamma_{4}^{\prime}\right)^{*}$ has integer scalar product with $\alpha_{2}$ (in order to be compatible with $\widehat{\alpha}_{2}^{\prime}$ ) and with the Wilson line $\mathbf{A}_{1}$. As

$$
\left(\gamma_{4}^{\prime}\right)^{*} \cdot \alpha_{2}=\sum_{A=1}^{7} C\left(A_{7}\right)_{4 A}^{-1}\left(\gamma_{A}^{\prime} \cdot \alpha_{2}\right)=C\left(A_{7}\right)_{42}^{-1}(-1)=\frac{8}{8}(-1)=-1 \in \mathbf{Z},
$$

it depends only on the choice of the Wilson line. The standard choice $\left(\gamma_{7}^{\prime}\right)^{*}$ gives

$$
\mathbf{A}_{1} \cdot\left(\gamma_{4}^{\prime}\right)^{*}=\left(\gamma_{7}^{\prime}\right)^{*} \cdot\left(\gamma_{4}^{\prime}\right)^{*}=C\left(A_{7}\right)_{74}^{-1}=\frac{4}{8} \notin \mathbf{Z}
$$

and the symmetry algebra is only $A_{7} \oplus A_{1}$.

An example of symmetry enhancement is provided by the choice $\mathbf{A}_{1}=$ $2\left(\gamma_{7}^{\prime}\right)^{*}$. This projects out the second root of $E_{8}$, because

$$
2\left(\gamma_{7}^{\prime}\right)^{*} \cdot \alpha_{2}=2 C\left(A_{7}\right)_{72}^{-1}(-1)=2 \frac{2}{8}=\frac{1}{2} \notin \mathbf{Z}
$$

but is compatible with the extra root $-\left(\gamma_{4}^{\prime}\right)^{*}$ :

$$
2\left(\gamma_{7}^{\prime}\right)^{*} \cdot\left(\gamma_{4}^{\prime}\right)^{*}=2 \frac{4}{8}=1 \in \mathbf{Z}
$$

In this case the gauge Lie algebra is $E_{7} \oplus A_{1}$.

\section{One parameter families}

By now we know a discrete set of critical values of the Wilson line, corresponding to maximal rank semisimple subalgebras of $\mathbf{g}$. In order to study the behaviour between these special points in the moduli space consider the one parameter families of Wilson lines defined by

$$
\mathbf{A}_{1}=p \beta_{I}^{*}, \quad p \in \mathbf{R}
$$

which interpolate between the model without background fields and the models with the standard Wilson lines $\beta_{I}^{*}$ corresponding to the removal of the

\footnotetext{
${ }^{14}$ This means that $-\left(\gamma_{4}^{\prime}\right)^{*}, \gamma_{2}^{\prime}, \ldots, \gamma_{7}^{\prime}$ are a system of simple roots of $E_{7}$.
} 
I-th dot from the extended Dynkin diagram of $\mathbf{g}$. The basis vectors of the lattice $\Gamma(p)$ corresponding to this choice of Wilson line are

$$
\begin{gathered}
\overline{\mathbf{k}}_{1}^{\prime}=\left(p \beta_{I}^{*},-\frac{1}{2} p^{2}\left(\beta_{I}^{*} \cdot \beta_{I}^{*}\right) \frac{1}{2} \mathbf{e}^{* 1}+\mathbf{e}_{1} ;-\frac{1}{2} p^{2}\left(\beta_{I}^{*} \cdot \beta_{I}^{*}\right) \frac{1}{2} \mathbf{e}^{* 1}-\mathbf{e}_{1}\right) \\
\widehat{\alpha}_{I}^{\prime}=\left(\alpha_{I}, \frac{p}{k^{I}} \frac{1}{2} \mathbf{e}^{* 1} ; \frac{p}{k^{I}} \frac{1}{2} \mathbf{e}^{* 1}\right)=\widehat{\alpha}_{I}+\frac{p}{k^{I}} \mathbf{k}^{1} \\
\widehat{\alpha}_{A}^{\prime}=\left(\alpha_{A}, \mathbf{0} ; \mathbf{0}\right)=\widehat{\alpha}_{A}, A \neq I \\
\widehat{\alpha}_{h}^{\prime}=\left(\alpha_{h}, p \frac{1}{2} \mathbf{e}^{* 1} ; p \frac{1}{2} \mathbf{e}^{* 1}\right)=\widehat{\alpha}_{h}+p \mathbf{k}^{1}
\end{gathered}
$$

We can now analyze the root system of this lattice for different values of $p$, using the methods introduced in the last sections.

For $p \notin \mathbf{Z}$, neither $\widehat{\alpha}_{I}$ nor $\widehat{\alpha}_{h}$ are in $\Gamma(p)$ and the gauge algebra is broken to

$$
\mathbf{g}(p)=\mathbf{g}_{I}^{(15)} \oplus u(1)
$$

where $\mathbf{g}_{I}^{(15)}$ is the rank 15 semisimple Lie algebra constructed by removing the I-th dot from the Dynkin diagram of $\mathbf{g}$ and $u(1)$ is the one dimensional abelian Lie algebra. (These Wilson lines solve (23) and (24), but not (25.)

The case $p=1$ corresponds to the standard solution (33) and the gauge algebra is the rank 16 semisimple Lie algebra $\mathbf{g}_{I}^{(16)}$ constructed by removing the I-th dot from the extended Dynkin diagram of $\mathbf{g}$ :

$$
\mathbf{g}(1)=\mathbf{g}_{I}^{(16)}
$$

If $p=k^{I}$, both $\widehat{\alpha}_{I}$ and $\widehat{\alpha}_{h}$ are in $\Gamma(p)$ :

$$
\mathbf{g}\left(k^{I}\right)=\mathbf{g}
$$

This also applies to all integer multiples of $k^{I}$. The lattices $\Gamma=\Gamma(0)$ and $\Gamma\left(k^{I}\right)$ are equal if and only if $\alpha_{I}^{*} \in \Gamma_{R}(\mathbf{g})$. Since $\left.\alpha_{I}^{*}=-k^{I} \beta_{I}^{*} \in \Gamma_{W}(\mathbf{g})\right)^{\top}$, there must be some integer $M \in \mathbf{Z}$ such that $M \alpha_{I}^{*} \in \Gamma_{R}(\mathbf{g})$ :

$$
\mathbf{g}\left(n k^{I}\right)=\mathbf{g}, \quad(\forall n \in \mathbf{Z}), \quad \Gamma\left(M k^{I}\right)=\Gamma,\left(\exists M \in \mathbf{Z}^{+}\right)
$$

\footnotetext{
${ }^{15}$ By construction $\beta_{I}^{*} \cdot \alpha_{I}=-\frac{1}{k^{I}}$ and $\beta_{I}^{*} \cdot \alpha_{J}=0$, for all $J \neq I$, implying $\beta_{I}^{*}=-\frac{1}{k^{I}} \alpha_{I}^{*}$.
} 
For all integer values of $p$, that are not multiples of $k^{I}$ the gauge Lie algebra

is $\mathbf{g}_{I}^{(16)}$. Whether $\Gamma(p)$ equals $\Gamma(1)$ or not depends on $\beta_{I}^{*} \cdot \beta_{I}^{*}$ being integer or not.

For all non integer values of $p$ the rank of the semisimple part is reduced by one. We could now study the 15-dimensional sublattice and add a discrete piece to the Wilson line to break $\mathbf{g}^{(15)}$ to its maximal rank subalgebras, then again reduce the rank and so on. The reduction of the rank will of cause increase the number of continuous parameters accordingly. By removing in all possible ways simple roots from all maximal rank semisimple subalgebras of $\mathbf{g}$, we can realize all subalgebras of the type semisimple $\oplus$ abelian.

We could also study one parameter families interpolating between $\mathbf{g}$ and its diverse subalgebras, investigate multiparameter families and so on. One example of a two parameter family will be discussed later after generalizing to general background fields. All these considerations give some information about the Narain moduli space that can be made more and more precise by investing time and effort.

\section{Enhancement of symmetry by one Wilson line}

By a "critical" or "multicritical" Wilson line people usually mean values of the Wilson line that (in the Narain model) extend the rank of the semisimple part of the gauge algebra beyond 16. This has not yet been analyzed systematically but there is the example of Ginsparg [8] that shows how $D_{16}$ can be enlarged to $D_{17}$ (or more generally to $D_{16+d}$, if $\geq d$ dimensions are compactified) by a suitable choice of Wilson line(s). I will now discuss a more general problem but restrict the analysis for the moment to lattices of the type $\Gamma_{17 ; 1} \oplus \Gamma_{5 ; 5}$ that means to the case of one nonvanishing Wilson line.

Suppose that the Wilson line is chosen such that the lattice contains $l \leq 16$ roots. We can then look for a vector of the form

$$
\widehat{\alpha}_{l+1}=(\mathbf{v}, \mathbf{w} ; \mathbf{0})
$$

which extends the root system. Then the $l+1$ simple root vectors must fulfill

$$
\widehat{\alpha}_{A} \cdot \widehat{\alpha}_{B}=C_{A B}^{(l+1)}, \quad A, B=1, \ldots, l+1,
$$


where $C_{A B}^{(l+1)}$ is the Cartan matrix of a rank $l+1$ semisimple Lie algebra. By inspection of the basis, the most general ansatz for $\widehat{\alpha}_{l+1}$ is

$$
\begin{gathered}
\widehat{\alpha}_{l+1}=\overline{\mathbf{k}}_{1}+m \mathbf{k}^{1}, m \in \mathbf{Z} \\
=\left(\mathbf{A}_{1},-\frac{1}{2}\left(\mathbf{A}_{1} \cdot \mathbf{A}_{1}\right) \frac{1}{2} \mathbf{e}^{* 1}+\mathbf{e}_{1} ;-\frac{1}{2}\left(\mathbf{A}_{1} \cdot \mathbf{A}_{1}\right) \frac{1}{2} \mathbf{e}^{* 1}-\mathbf{e}_{1}\right)+m\left(\mathbf{0}, \frac{1}{2} \mathbf{e}^{* 1} ; \frac{1}{2} \mathbf{e}^{* 1}\right)
\end{gathered}
$$

Since our space-time torus is $\mathbf{R}^{d} / 2 \pi \Lambda$, the square root of $\mathbf{e}_{1} \cdot \mathbf{e}_{1}$ is the "radius" $\square$ of the 1-direction, which we have chosen to be orthogonal to the others. This implies:

$$
\mathbf{e}_{1} \cdot \mathbf{e}_{1}=R^{2}, \quad \mathbf{e}^{* 1} \cdot \mathbf{e}^{* 1}=\frac{1}{R^{2}}, \quad \mathbf{e}_{1}=R^{2} \mathbf{e}^{* 1}
$$

It is very useful to rewrite $\overline{\mathbf{k}}_{1}^{\prime}$ :

$$
\overline{\mathbf{k}}_{1}^{\prime}=\left(\mathbf{A}_{1}, 2 \mathbf{e}_{1}+D \frac{1}{2} \mathbf{e}^{* 1} ; D \frac{1}{2} \mathbf{e}^{* 1}\right), \quad D=2\left(-R^{2}-\frac{1}{4}\left(\mathbf{A}_{1} \cdot \mathbf{A}_{1}\right)\right)
$$

This shows that $\widehat{\alpha}_{l+1}$ can be in $\Gamma^{\prime}$ only if

$$
D=-m \in \mathbf{Z}
$$

The extra root has the form

$$
\widehat{\alpha}_{l+1}=\left(\mathbf{A}_{1}, 2 \mathbf{e}_{1} ; \mathbf{0}\right)
$$

$\widehat{\alpha}_{l+1}$ being a root vector also implies:

$$
\widehat{\alpha}_{l+1} \cdot \widehat{\alpha}_{l+1}=\mathbf{A}_{1} \cdot \mathbf{A}_{1}+4 \mathbf{e}_{1} \cdot \mathbf{e}_{1}=2
$$

(85) and (87) imply two conditions:

$$
\begin{gathered}
2 R^{2}+\frac{1}{2} \mathbf{A}_{1} \cdot \mathbf{A}_{1}=m \in \mathbf{Z} \\
4 R^{2}+\mathbf{A}_{1} \cdot \mathbf{A}_{1}=2
\end{gathered}
$$

\footnotetext{
${ }^{16}$ The geometric interpretation is ambiguous due to duality transformations which map $R$ tp $\frac{1}{2 R}$, but leave the physics of the model invariant [9], 10].
} 
Combinig them fixes $m=1$ and gives a relation between the radius of the 1-direction of the torus and the norm of the Wilson line:

$$
R^{2}=\frac{1}{2}-\frac{1}{4} \mathbf{A}_{1} \cdot \mathbf{A}_{1}
$$

This shows that the enhancement of symmetry takes only place for special values of the radius. Since $R^{2}>0$ and $\mathbf{A}_{1} \cdot \mathbf{A}_{1} \geq 0$ we have the following bounds on the radius and the norm of the Wilson line:

$$
0<R \leq \frac{1}{2} \sqrt{2}, \quad 0 \leq \mathbf{A}_{1} \cdot \mathbf{A}_{1} \leq 2
$$

The restriction on the radius-parameter is afflicted by the ambiguity of geometrical interpretation due to duality (or target space modular) transformations [10], [9]. As the radius of the transformed model is $1 / 2 R$ we cannot distinguish between very small and very large radii and the inequality above only excludes copies.

In order to analyze the condition (81) we assume that the extra root extends the rank $l$ algebra such that it has a nonvanishing scalar product with only one of the 1 simple roots, let us say with the I-th one:

$$
\widehat{\alpha}_{l+1} \cdot \widehat{\alpha}_{A}=\mathbf{A}_{1} \cdot \alpha_{A}=-\delta_{A I} \Rightarrow \mathbf{A}_{1}=-\alpha_{I}^{*}+\mathbf{v}
$$

where $\mathbf{v}$ is in the orthogonal complement (with respect to $\mathbf{R}^{16}$ ) of the linear hull of $\alpha_{1}, \ldots, \alpha_{l}$. If the extra root has vanishing scalar product with all other roots, $\mathbf{A}_{1}$ lies completely in this complement and the gauge algebra is extended by a $A_{1}$ algebra. For $\mathbf{A}_{1}=0$ we have $R=1 / \sqrt{2}$. This is well known from one dimensional compactifications [13] and $(c=1)-\mathrm{CFT}$ [9]. If $\mathbf{A}_{1}$ shall have a nonvanishing scalar product with more than one other root it must be an appropriate linear combination of fundamental weights plus an arbitrary orthogonal piece.

Finally note that we now have found examples of special values for all the 17 continuous parameters (moduli) of $\Gamma_{17 ; 1}$, the Wilson line $\mathbf{A}_{1}$ and the radius $R$.

\section{Symmetry breaking and enhancement by more then one Wilson line}

In this section we return to the general case by allowing arbritray background fields. In this case all the $6+6$ dimensions of the momentum lattice that come 
from compactification (the compactification sector) can be mixed with the 16 dimensions coming from the gauge sector of the ten-dimensional theory. The most obvious generalization of the previous results is to split the role of the single Wilson line used above among the up to six Wilson lines. As it was possible to construct arbitrary subalgebras by one Wilson line this cannot lead to new symmetry algebras but only to different spectra. Instead of studying this pure refinement of the old pattern we will investigate the new patterns of symmetry breaking and enhancement that are now available: As a first step we will show that extra roots can be constructed for special domains of values of the Wilson lines. The effect on the gauge algebra coming from the 16-dimensional sector will be investigated in a second step. The following parametrization of the basis vectors of the momentum lattice makes the appearance of extra roots very transparent:

$$
\begin{gathered}
\mathbf{k}^{\prime i}=\left(0, \frac{1}{2} \mathbf{e}^{* i} ; \frac{1}{2} \mathbf{e}^{* i}\right)=\mathbf{k}^{i} \\
\overline{\mathbf{k}}_{i}^{\prime}=\left(\mathbf{A}_{i}, 2 \mathbf{e}_{i}+D_{i j} \frac{1}{2} \mathbf{e}^{* j} ; D_{i j} \frac{1}{2} \mathbf{e}^{* j}\right) \\
l_{A}^{\prime}=\left(\mathbf{e}_{A},-\left(\mathbf{e}_{A} \cdot \mathbf{A}_{k}\right) \frac{1}{2} \mathbf{e}^{* k} ;-\left(\mathbf{e}_{A} \cdot \mathbf{A}_{k}\right) \frac{1}{2} \mathbf{e}^{* k}\right)
\end{gathered}
$$

where

$$
D_{i j}=2\left(B_{i j}-G_{i j}-\frac{1}{4}\left(\mathbf{A}_{i} \cdot \mathbf{A}_{j}\right)\right)
$$

By inspection the only way to construct root vectors out of the $\mathbf{k}^{\prime}$ and the $\overline{\mathbf{k}}_{i}^{\prime}$ is to take as candidates for simple roots the linear combinations

$$
\widehat{\alpha}_{16+i} \equiv \overline{\mathbf{k}}_{i}^{\prime}-D_{i j} \mathbf{k}^{\prime j}=\left(\mathbf{A}_{i}, 2 \mathbf{e}_{i} ; \mathbf{0}\right)
$$

and these are in the momentum lattice $\Gamma^{\prime}$ if and only if

$$
D_{i j} \in \mathbf{Z} \text {. }
$$

Note that this condition only fixes a combination of the Wilson lines and the six-dimensional lattice metric $G_{i j}$ which is the symmetric part of $D_{i j}$ and leaves us free to vary one of these objects if we vary the other accordingly. The extra roots form the system of simple roots of a semisimple Lie algebra of rank $\leq 6$, if

$$
\widehat{\alpha}_{16+i} \cdot \widehat{\alpha}_{16+j}=\mathbf{A}_{i} \cdot \mathbf{A}_{j}+4 G_{i j}=C_{i j}
$$


where $C_{i j}$ is the Cartan matrix of such an algebra. In this equation the matrices in the middle are subject to constraints: $G_{i j}$ must be positive definite because it is a metric and $\mathbf{A}_{i} \cdot \mathbf{A}_{j}$ must be positive semidefinite because it is a matrix of scalar products of vectors. This implies for example that there are no admissible solutions to equation (99) when one of the Wilson lines has a norm squared $\geq 2$ because then $G_{i j}$ would have a zero or negative eigenvalue. We have also to ensure that $D_{i j}$ is an integer because this is necessary and sufficient for the extra roots being vectors in $\Gamma^{\prime}$. But combining (96) and (99) this is equivalent to

$$
B_{i j}=G_{i j}+\frac{1}{4} \mathbf{A}_{i} \cdot \mathbf{A}_{j}=\frac{1}{4} C_{i j} \text { modulo } \frac{1}{2}
$$

This condition on $B_{i j}$ can always be fulfilled although $B_{i j}$ is antisymmetric: The Cartan matrices $C_{i j}$ of simply laced Lie algebras have entries 2 on the diagonal and 0 and -1 off diagonal and they are symmetric so that they are also antisymmetric modulo 2 . If one sets all Wilson lines to zero one recovers the old results [8] about symmetry enhancement by the $B_{i j}$ field. On the other hand we can set $B_{i j}$ to zero and analyze the possibility to construct extra roots through the Wilson lines and the lattice metric alone. But if we want to have (99) and $D_{i j} \in \mathbf{Z}$ the Cartan matrix cannot have -1 's as offdiagonal entries and the extra roots are restricted to form a Lie algebra that is simply a direct sum of $A_{1}$-algebras. Combining nonvanishing background fields of all kinds we can have symmetry breaking by Wilson lines and symmetry enhancement by the $B_{i j}$-field and by Wilson lines. If we construct extra roots (97) with nonvanishing Wilson lines then they can have integer scalar products with some roots from the 16-dimensional sector, resulting in a simple summand of the total gauge algebra that is made of roots coming from both sectors.

Each choice of Wilson lines will break the gauge algebra $\mathbf{g}^{(16)}$ of the sixteen dimensional sector to a subalgebra. In fact a root of $\Gamma$ of the form

$$
\widehat{\alpha}=(\alpha, \mathbf{0} ; \mathbf{0})
$$

is in the new lattice if and only if

$$
\alpha \cdot \mathbf{A}_{i} \in \mathbf{Z} \quad(i=1, \ldots, 6)
$$

because the momentum lattice is selfdual. Of course the Wilson lines that produce extra roots in the six-dimensional sector need not to fulfill the criterion for breaking $\mathbf{g}^{(16)}$ to a maximal rank subalgebra and thus will generically 
reduce the rank of $\mathbf{g}^{(16)}$. Let us analyze the situation by an example that is typical for all models with 2 nonvanishing Wilson lines:

Example: We start with $\mathbf{g}^{(16)}=D_{16}$ and take two nonvanishing Wilson lines, $\mathbf{A}_{1}$ and $\mathbf{A}_{2}$. The metric $G_{i j}$ is chosen to be blockdiagonal, such that the two directions to which the nonvanishing Wilson lines are assigned are orthogonal to the other four directions that can therefore be ignored. We can then construct the two simply laced semisimple Lie algebras $A_{1} \oplus A_{1}$ and $A_{2}$ by setting $B_{i j}$ to its critical value (100) and solving (99) for

$$
C_{i j}^{\left(A_{1} \oplus A_{1}\right)}=\left(\begin{array}{ll}
2 & 0 \\
0 & 2
\end{array}\right) \text { or } C_{i j}^{\left(A_{2}\right)}=\left(\begin{array}{cc}
2 & -1 \\
-1 & 2
\end{array}\right)
$$

Both cases can be treated simultaneously. Next we try to use our freedom in choosing $\mathbf{A}_{1}$ and $\mathbf{A}_{2}$ to break $D_{16}$ to the subalgebra $A_{3} \oplus A_{3} \oplus D_{10}$. This subalgebra is constructed by removing first the third root from the extended Dynkin diagram of $D_{16}$, resulting in the maximal subalgebra $A_{3} \oplus D_{13}$ and then removing the third root from the extended Dynkin diagram of the $D_{13}$. If we denote by $\gamma_{1}, \ldots, \gamma_{3}, \gamma_{4}, \ldots, \gamma_{6}, \gamma_{7}, \ldots, \gamma_{16}$ the simple roots of the $A_{3} \oplus$ $A_{3} \oplus D_{10}$ subalgebra then one solution of this symmetry breaking problem is given by

$$
\mathbf{A}_{1}=\gamma_{3}^{*}, \quad \mathbf{A}_{2}=\gamma_{6}^{*}
$$

Substituting the scalar products

$$
\begin{aligned}
& \mathbf{A}_{1} \cdot \mathbf{A}_{1}=\gamma_{3}^{*} \cdot \gamma_{3}^{*}=\frac{3}{4} \\
& \mathbf{A}_{2} \cdot \mathbf{A}_{2}=\gamma_{6}^{*} \cdot \gamma_{6}^{*}=\frac{3}{4}
\end{aligned}
$$

and

$$
\mathbf{A}_{1} \cdot \mathbf{A}_{2}=\gamma_{3}^{*} \cdot \gamma_{6}^{*}=0
$$

into equation (99) we find that we have to choose

$$
\left(G_{i j}\right)_{i, j=1}^{2}=\frac{1}{16}\left(\begin{array}{cc}
5 & 0 \\
0 & 5
\end{array}\right)
$$

and

$$
\left(G_{i j}\right)_{i, j=1}^{2}=\frac{1}{16}\left(\begin{array}{cc}
5 & -4 \\
-4 & 5
\end{array}\right)
$$


Both solution are positive definite and therefore admissible. Calculating the scalar products between the extra simple roots

$$
\begin{aligned}
& \widehat{\gamma}_{17}=-\left(\gamma_{3}^{*}, 2 \mathbf{e}_{1} ; 0\right) \\
& \widehat{\gamma}_{18}=-\left(\gamma_{6}^{*}, 2 \mathbf{e}_{2} ; 0\right)
\end{aligned}
$$

and the simple roots $\widehat{\gamma}_{A}, A=1, \ldots, 16$ of $A_{3} \oplus A_{3} \oplus D_{10}$

$$
\begin{aligned}
& \widehat{\gamma}_{A} \cdot \widehat{\gamma}_{17}=-\gamma_{A} \cdot \gamma_{3}^{*}=-\delta_{A 3} \\
& \widehat{\gamma}_{A} \cdot \widehat{\gamma}_{18}=-\gamma_{A} \cdot \gamma_{6}^{*}=-\delta_{A 6}
\end{aligned}
$$

we find that these roots are linked together such that the total symmetry algebra is $A_{4} \oplus A_{4} \oplus D_{10}$ in the first and $A_{8} \oplus D_{10}$ in the second case. This example shows how symmetry breaking and symmetry enhancement can interfere. Note that the semisimple part of the new Lie algebra that has a bigger rank then the one we started with although we did not simply extend the original algebra by simple roots. We have also shown that one can construct "big" simple pieces, like $A_{4}$ and $A_{8}$ by combining roots from the gauge sector and the compactification sector.

We can now study two parameter families of theories by setting

$$
\mathbf{A}_{1}=p_{1} \gamma_{3}^{*}, \quad \mathbf{A}_{2}=p_{2} \gamma_{6}^{*}
$$

and varying the $p_{i}$. If both $p_{i} \notin \mathbf{Z}$ the roots $\gamma_{3}$ and $\gamma_{6}$ are projected out and the symmetry is reduced to

$$
A_{2} \oplus A_{2} \oplus D_{10} \oplus\left\{\begin{array}{l}
A_{1} \oplus A_{1} \\
A_{2}
\end{array}\right.
$$

respectively, that is we get critical surfaces of rank 16 models in the Narain moduli space. If we restrict one $p_{i}$ to be an integer we get critical lines of rank 17 models within these surfaces. These lines intersect each other in critical points representing rank 18 models when both $p_{i}$ are integers. We can try to learn about the global geometry of this surface by analyzing the constraint that the metric $G_{i j}$ must be positive definite. This fixes the domain of the parameters to be

$$
0<p_{i}^{2}<\frac{8}{3}, \quad i=1,2
$$


in the $A_{1} \oplus A_{1}$ case and to be

$$
0<p_{1}^{2}<\frac{8}{3} \text { and }\left(2-\frac{3}{4} p_{1}^{2}\right)\left(2-\frac{3}{4} p_{2}^{2}\right)-1>0
$$

in the $A_{2}$ case.

Although we analyzed a specific example it is clear that the pattern found here is generic for models with more then one Wilson line. One interesting generalization of this example is to take the space-time torus to be a product of three two-dimensional tori, where each of these is proportional to the maximal torus of $S U(3)$ and six Wilson lines. This region of the Narain moduli space is the natural starting point for the construction of $\mathbf{Z}_{3}$-orbifolds with continuous Wilson lines. (Of course the background fields must be further constraint, so that the orbifold twist is an automorphism of the momentum lattice. This and related questions are under investigation.)

The example suggests the question whether it is possible to combine every symmetry breaking by Wilson lines with every system of extra roots generated by the $B_{i j}$ field. To give a counterexample, it is sufficient to study the simplest nontrivial case: Assume that there is one nonvanishing Wilson line $\mathbf{A}_{1}$ and that the extra root $\left(\mathbf{A}_{1}, 2 \mathbf{e}_{1} ; \mathbf{0}\right)$ has a nonvanishing scalar product with precisely one of the other extra roots $\left(\mathbf{0}, 2 \mathbf{e}_{i} ; \mathbf{0}\right), i=2, \ldots, l \leq 6$. Then the only nontrivial constraint is that the matrix

$$
G_{i j}=\frac{1}{4}\left(C_{i j}-\mathbf{A}_{i} \cdot \mathbf{A}_{j}\right)=\frac{1}{4}\left(\begin{array}{cccc}
2-\mathbf{a}^{2} & -1 & 0 & \cdots \\
-1 & 2 & * & \\
\vdots & * & \ddots & \\
& & & 2
\end{array}\right)
$$

must be positive definite, where $\mathbf{a}^{2}=\mathbf{A}_{1} \cdot \mathbf{A}_{1}>0$. The matrix that we get by removing the first row and the first column is the Cartan matrix of a Lie algebra and therefore positive definite. So we have only to look for the determinant of $G_{i j}$ and this can be done case by case: The Lie algebra $\mathbf{g}^{(6)}$ formed by the extra roots must be one of the Lie algebras

$$
A_{l} \oplus X_{6-l}, \quad 1 \leq l \leq 6, \quad D_{l} \oplus X_{6-l}, \quad 4 \leq l \leq 6, \quad E_{6}
$$

where the ideal $X_{6-l}$ is orthogonal to the ideal to which the first root belongs. The determinant of $G_{i j}$ can be expanded and expressed as a function of $\mathbf{a}^{2}$ 
and the determinants of the Cartan matrices of the subalgebras of $\mathbf{g}^{(6)}$. The result is an upper bound on $\mathbf{a}^{2}$

$$
\operatorname{det}\left(G_{i j}\right)>0 \Leftrightarrow \mathbf{a}^{2}<\left\{\begin{array}{cc}
\frac{l+1}{l}, & \mathbf{g}^{(6)}=A_{l} \oplus X_{6-l} \\
1, & \mathbf{g}^{(6)}=D_{l} \oplus X_{6-l} \\
\frac{13}{4}, & \mathbf{g}^{(6)}=E_{6}
\end{array}\right.
$$

These bounds must be compared to the minimum length that the Wilson line needs in order to break $\mathbf{g}^{(16)}$ to a given subalgebra. For all subalgebras that can be constructed by removing one dot from the extended Dynkin diagram this length can be calculated very simply by looking at the simple ideal of the subalgebra to which the highest root of $\mathbf{g}^{(16)}$ belongs. The standard Wilson line is then the dual of this vector with respect to the subalgebra and its length can be read off the inverse Cartan matrix of the subalgebra. The highest root can be part of a A-type Lie algebra or of a D-type Lie algebra. In the first case it is the first or last root of this Lie algebra and has a norm $<1$ : Equation (120) can then be fulfilled for arbitrary $\mathbf{g}^{(6)}$. In the second case the highest root of $\mathbf{g}^{(16)}$ is always the last or (equivalently) the next to last root of the D-type subalgebra. Then $\mathbf{a}^{2} \geq 1$ if the the rank of the summand is $\geq 4$. One can then look for nonstandard solutions of the symmetry breaking problem. For example the difference of the last and first to last root is such a solution with norm squared 1. As the shortest weight vectors of $D_{n}$ Lie algebras have length 1 shorter solutions cannot exist. We conclude that this case of symmetry breaking is incompatible with an $\mathbf{g}^{(6)}$ of the type $D_{l} \oplus X_{6-l}$. This shows that even in the case of one single Wilson line incompatible patterns of extended and broken symmetry do exist.

\section{The shift vector realization}

The shift vector method [23], [18] is, like our construction of critical Wilson lines a procedure that starting from one model produces a discrete set of models and makes it possible to project out or bring in some states. Therefore it is natural to ask for the relation between the two methods. Although the shift vector method was mostly used in covariant lattice theories we can use it in our context because all Narain lattices can be embedded in covariant lattices and if we restrict the shift vector to operate only on the Narain part we can stay inside the Narain model. The analysis will be done for critical 
Wilson lines that only exchange two basis vectors; this includes all breakings to maximal subalgebras.

The essentials of the shift vector method are the following: We start with some given even selfdual lorentzian lattice $\Gamma \equiv \Gamma_{p ; q}$ and choose some vector $\mathbf{s} \notin \Gamma$, such that $N \mathbf{s} \in \Gamma$, for some minimal positive integer $N$. s defines the "invariant sublattice"

$$
\Gamma_{0}=\{\mathbf{v} \in \Gamma \mid \mathbf{v} \cdot \mathbf{s} \in \mathbf{Z}\} .
$$

If $\mathbf{s}^{2}=2 m / N$, for some $m \in \mathbf{Z}$, the lattice

$$
\widetilde{\Gamma}=\sum_{k=0}^{N-1}\left(\Gamma_{0}+k \mathbf{s}\right)
$$

is even selfdual if and only if $\Gamma$ is. Shift vectors can be applied successively in order to project out unwanted or to bring in wanted states (more properly: conjugacy classes of lattice vectors modulo the underlying root lattice). Successive shift vectors are compatible, that is the ordering is irrelevant, if and only if their scalar product is an integer. All compatible shift vectors are then elements of the new lattice.

Comparing this prescription to the relation between the two basis (16) and (18) connected by the switching on of background fields it is natural to assume that the new basis vectors (those basis vectors of $\Gamma^{\prime}$ that are not in $\Gamma$ ) can be interpreted as successive shift vectors. Of course all new basis vectors must be rational linear combinations of the old ones so that some multiple of each new basis vector is in the old lattice. This can be achieved by allowing only rational values for the background fields. Then the new basis vectors are admissible and compatible shift vectors because they all have even norm squared and mutual integer scalar product by construction (see (17)). What must be proven is that the lattices $\widetilde{\Gamma}$, constructed by the successive application of the new basis vectors as shift vectors and $\Gamma^{\prime}$, generated by (18) are identical. All basis vectors of $\Gamma^{\prime}$ are in $\widetilde{\Gamma}$ because they are either shift vectors or invariant vectors of the old lattice $\Gamma$, implying:

$$
\Gamma^{\prime} \subset \widetilde{\Gamma}
$$

Dualizing this equation gives

$$
\left(\Gamma^{\prime}\right)^{*} \supset(\widetilde{\Gamma})^{*}
$$


But both lattices are are selfdual and so we have the other inclusion and thus equality.

This result shows how one can keep the information about continuous parameters when working with shift vectors. If one is only interested in the gauge group it is preferable to work with a basis instead of the complete list of conjugacy classes as is usually done when applying shift vectors [18]. The full information can be restored, if needed, by adding the basis vectors modulo the root lattice of the underlying Lie algebra, until the full group of conjugacy classes of the lattice [18] is generated.

\section{Further applications}

The results and techniques established here do not only enlarge the knowledge about toroidal compactifications but will also be helpful in more general situations. One future application is the construction of toroidal orbifolds with continuous Wilson lines, which is possible when the twist is realized as a rotation both in the compactification and in the gauge sector [17], [15]. Using the parametrization of general background fields introduced here I have been able until now to bring the compatibility condition between a symmetric twist and general background fields in a simple form and to calculate the number of untwisted moduli. Combining the shift vector realization of critical background fields with the results of [23] one should be able to reinterpret covariant lattice models as orbifolds with certain critical background fields. The usual formalism for orbifolds in which the twist is realized as a shift [15] in the gauge sector allows only for discrete Wilson lines and can be interpreted as a hybrid construction because it uses both rotations and shift vectors. Some of them may therefore also be critical points ( or hypersurfaces ) in the moduli space of the bigger class of orbifolds with continuous Wilson lines. I hope that the methods developed here will help to analyze these questions further.

\section{References}

[1] R. N. Cahn, Semi Simple Lie Algebras and Their Representations. Benjamin and Cummings (1984). 
[2] J. F. Cornwell, Group Theory in Physics. Academic Press (1984).

[3] R. Dijkgraaf, E. Verlinde, and H. Verlinde, $c=1$ Conformal Field Theory on Riemann Surfaces. Comm. Math. Phys. 115 (1988) 649.

[4] L. Dixon, P. Ginsparg, and J. Harvey, $\hat{c}=1$ Superconformal Field Theory. Nucl. Phys. B 306 (1988) 470.

[5] L. Dixon, J. Harvey, C. Vafa, and E. Witten, Strings on Orbifolds. Nucl. Phys. B 261 (1985) 678.

[6] L. Dixon, J. Harvey, C. Vafa, and E. Witten, Strings on Orbifolds (II). Nucl. Phys. B 274 (1986) 285.

[7] S. Ferrara, and S. Theisen, Moduli Spaces, Effective Actions and Duality Symmetry in String Compactifications. CERNTH.5652/90.

[8] P. Ginsparg, On toroidal compactification of heterotic superstrings. Phys. Rev. D 38 (1987) 648.

[9] P. Ginsparg, Applied Conformal Field Theory. HUTP-88/AO54.

[10] A. Giveon, E. Rabinovici, and G. Veneziano, Duality in String Background Spaces. Nucl. Phys. B 322 (1989) 167.

[11] P. Goddard and D. Olive, Kac-Moody and Virasoro Algebras in Relation to Quantum Physics. Int. J. Mod. Phys. A1 (1986) 303.

[12] M. Golubitsky and B. Rothschild, Primitive Subalgebras of Exceptional Lie Algebras. Bull. Amer. Math .Soc. 77 (1971) 983.

[13] M. Green, J. Schwarz and E. Witten, Superstring Theory. (2 volumes). Cambridge University Press (1987).

[14] J. E. Humphreys, Introduction to Lie Algebras and Representation Theory. Springer (1972).

[15] L. E. Ibanez, J. Mas, H. P. Nilles and F. Quevedo, Heterotic Strings in Symmetric and Asymmetric Orbifold Backgrounds. Nucl.Phys. B 301 (1988) 157. 
[16] L. E. Ibanez, The Search for a Standard Model $S U(3) \times S U(2)$ $\times U(1)$ Superstring. CERN-Th.4769/87.

[17] L. E. Ibanez, H. P. Nilles and F. Quevedo, Reducing the Rank of the Gauge Group in Orbifold Compactifications of heterotic string. Phys. Lett. B192 (1987) 332.

[18] W. Lerche, A. N. Schellekens and N. P. Warner, Lattices and Strings. Phys. Rep. 177 (1989) 1.

[19] M. Lorente and B. Gruber, Classification of Semisimple Subalgebras of Simple Lie Algebras. J. Math. Phys. 13 (1972) 1639.

[20] K. Narain, M. Sarmadi and E. Witten, A Note on Toroidal Compactification of Heterotic String Theory. Nucl. Phys. B 279 (1987) 369.

[21] K. Narain, New heterotic string theories in uncompactified dimensions < 10. Phys. Lett. 169 b (1986) 41.

[22] K. Narain, M. Sarmadi and C. Vafa, Asymmetric Orbifolds. Nucl. Phys. B 288 (1987) 551.

[23] A. N. Schellekens and N. P. Warner, Weyl-Groups, Supercurrents and Covariant Lattices Nucl. Phys. B 308 (1988) 397.

[24] A. Shapere and F. Wilczek, Self Dual Models with Theta Terms. Nucl. Phys. B 320 (1989) 669. 